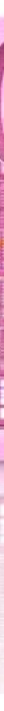

\title{
Leitlinien in der Neurologie: Wer bestellt, der zahlt (?)
}

Kein klinisch tätiger Neurologe ist noch in der Lage, das gesamte klinische neurologische Wissen vorzuhalten und daher jeden Patienten auf dem neuesten Stand des Wissens zu diagnostizieren und zu behandeln. Deshalb spielen diagnostische und therapeutische Leitlinien eine immense Rolle für den klinischen Alltag. Die Deutsche Gesellschaft für Neurologie (DGN) mit ihrer hohen Leitlinienkultur hat als erste Fachgesellschaft überhaupt fast alle wichtigen Krankheiten ihres Fachgebietes in Leitlinien dargestellt. Diese Leitlinien werden auch regelmäßig aktualisiert, wobei die Frequenz vom Zuwachs wissenschaftlicher Erkenntnisse abhängt. Die aufwändigsten Leitlinien sind S3-Leitlinien, von denen es in der Neurologie drei gibt, nämlich Sekundärprävention des Schlaganfalls, Demenzen und - neu das idiopathische Parkinson-Syndrom.

Die S3-Leitlinie idiopathisches Parkinson-Syndrom hat die DGN zusammen mit 29 anderen Fachgesellschaften erst unlängst fertiggestellt und die Langversion zur Kommentierung im Internet verfügbar gemacht. Die S3-Leitlinie Demenzen ist durch eine Kooperation der Deutschen Gesellschaft für Psychiatrie und Psychotherapie, Psychosomatik und Nervenheilkunde (DGPPN) mit der DGN entstanden. An dieser Leitlinie haben 24 Gesellschaften und Verbände mitgearbeitet. Die Veröffentlichung der Vorabversion gibt allen Mitgliedern der DGN die Möglichkeit, ihre Kommentare an die Sprecher der Steuerungsgruppe zu schicken. Nach Ablauf der entsprechenden Frist, werden die Leitlinien dann endgültig publiziert.

S3-Leitlinien sind unglaublich arbeitsaufwändig. Für jede der Fragestellungen muss eine systematische Literaturrecherche durchgeführt werden und die so identifizierten Studien, die bestimmten Qualitätscharakteristika genügen, müssen in DatenExtraktionsbögen übertragen werden. Diese Arbeit wird überwiegend von Biometrikern und Epidemiologen durchgeführt und diese Leistungen müssen von der DGN bezahlt werden. Alle anderen, die an der Erstellung von Leitlinien mitarbeiten, tun dies ehrenamtlich. Allerdings fallen auch Reisekosten für die verpflichtenden Treffen der Autorengruppen der Unterkapitel

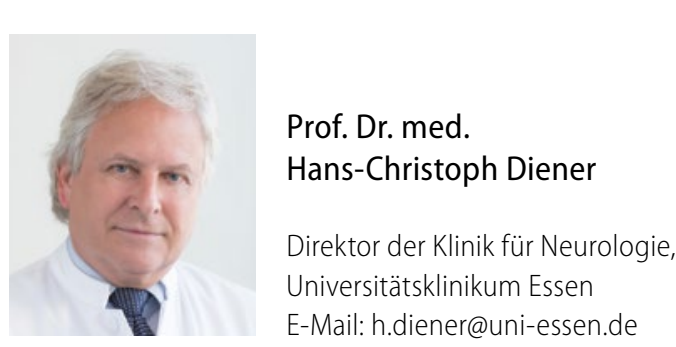

und der beteiligten Gesellschaften und Berufsverbände an. Alle diese Kosten werden von den Mitgliedern der DGN aus ihren Mitgliedsbeiträgen und den Erlösen des Jahreskongresses getragen.

Die Politik legt großen Wert auf die regelmäßige Erstellung und Aktualisierung von Leitlinien in der Medizin. Sie ist jedoch nicht bereit - wie etwa in England üblich - den erheblichen Aufwand für die Erstellung und Pflege von Leitlinien zu bezahlen. Das National Institute for Health and Care Excellence (NICE, https://www.nice.org.uk/) ist eine staatlich finanzierte Behörde, die im Auftrag der Regierung Leitlinien mithilfe von Experten und Methodikern erstellt. Diese Leitlinien sind qualitativ hochwertig und berücksichtigen nicht nur die wissenschaftliche Evidenz, sondern stellen auch Kosten-Nutzen-Analysen für das Gesundheitssystem zur Verfügung. In Deutschland gewinnt man den Eindruck, dass manche der vom Staat geschaffenen Behörden eher dem Zweck dienen, medizinische Innovationen zu bremsen und manche hochpreisige Therapien zu verhindern (was im Einzelfall bei einer ungünstigen Kosten-Nutzen-Analyse durchaus sinnvoll ist). Eine gute Investition des Staates wäre auch hier ein Institut, das für die häufigsten medizinischen Krankheitsbilder die Erstellung hochwertiger Leitlinien organisiert und finanziert. Daher sollte das althergebrachte Prinzip gelten: Wer bestellt, der zahlt. 